\title{
The value and role of non-invasive prenatal testing in a select South African population
}

\author{
C N Mnyani, ${ }^{1}$ BA, MB ChB, FCOG (SA); E Nicolaou, ${ }^{1,2}$ MD, FCOG (SA), Dip Fet Med; S Bister, ${ }^{2}$ RPN \\ ${ }^{1}$ Department of Obstetrics and Gynaecology, Faculty of Health Sciences, University of the Witwatersrand, Johannesburg, South Africa \\ ${ }^{2}$ Maternal and Fetal Medicine Centre, Morningside Mediclinic, Johannesburg, South Africa
}

Corresponding author: C N Mnyani (coceka.mnyani@wits.ac.za)

\begin{abstract}
Background. Concerns have been raised about the injudicious use of non-invasive prenatal testing (NIPT) using cell-free DNA (cfDNA), which often leads to inaccuracies in interpretation of the role and value of cfDNA in prenatal screening.

Objective. To determine the value and role of NIPT in a select South African (SA) population.

Methods. A retrospective review of patients who elected to have NIPT between 1 October 2013 and 30 June 2015 at the Morningside Mediclinic Maternal and Fetal Medicine Centre in Johannesburg, SA. Patients had NIPT after either combined first-trimester screening (CFTS) or a second-trimester ultrasound scan. Data were collected on details of the first- and/or second-trimester screening, results of the NIPT, invasive tests done, decisions made in the event of abnormal results, and pregnancy outcomes.

Results. Overall, 3473 first- and second-trimester fetal assessments were done at the centre during the study period, and $2.3 \%$ of patients ( $n=82)$ elected to have NIPT. The majority of these individuals elected to have NIPT on the basis of positive findings on CFTS, or markers of aneuploidy detected on a second-trimester ultrasound scan. Of the tests done, $97.6 \%$ produced results. Of those with no results, one did not meet quality metrics and the other had a low fetal fraction of cfDNA. There were two abnormal NIPT results, one indicating a high risk of trisomy 13 and the other a triploidy. Patients who screened negative elected not to have an invasive test.

Conclusion. The value of NIPT in this study was that it made it possible to avoid a number of invasive tests. NIPT had a role in contingency screening.
\end{abstract}

S Afr Med J 2016;106(10):1047-1050. DOI:10.7196/SAMJ.2016.v106i10.10650

Early and accurate diagnosis of fetal aneuploidies is one of the cornerstones of fetal medicine. While invasive tests - chorionic villus sampling (CVS), amniocentesis, or cordocentesis - remain the gold standard for diagnosis of aneuploidies, there have been several advances in non-invasive screening tests in both the first and second trimesters of pregnancy. Invasive testing carries a risk of miscarriage, albeit low in experienced hands, but devastating especially if the fetus is chromosomally normal, hence the need for developments in non-invasive tests. Detailed first- and second-trimester ultrasound scans, maternal characteristics and serum markers have been part of standard practice for aneuploidy screening for more than two decades. ${ }^{[1-3]}$ Non-invasive prenatal testing (NIPT) to screen for fetal aneuploidies by quantitative evaluation of the fetal fraction of cellfree DNA (cfDNA) in maternal blood is a relatively new technology that has advanced rapidly.

The presence of a fetal fraction of cfDNA in maternal blood was first described in $1997,{ }^{[4]}$ and it was reported that this represents the entire fetal genome and can be detected as early as the first trimester of pregnancy. There is abundant evidence to support the efficacy of NIPT, using cfDNA, in screening for trisomies 21 and 18 , and to a lesser extent trisomy $13 .{ }^{[5]}$ High efficacy has also been reported by test manufacturers for monosomy $\mathrm{X}$, sex chromosome trisomies and, for some tests, triploidy. As the technology advances more conditions are being tested for, including chromosomal microdeletions, and the fetal fraction of cfDNA is also being used to predict pregnancy complications such as pre-eclampsia. ${ }^{[6-8]}$

Concerns have been raised about the injudicious use of cfDNA testing, which often leads to inaccuracies in interpretation of the role and value of cfDNA in prenatal screening. ${ }^{[9,10]}$ As a result, several recommendations on the use of cfDNA for prenatal testing have been published. ${ }^{[9,11-13]}$ Adequate data on NIPT only exist for high- risk populations, i.e. pregnant women with a risk of chromosomal abnormalities of $>1: 100$; most guidelines therefore recommend NIPT use for this group of obstetric patients. ${ }^{[12,13]}$ Guidelines also emphasise the importance of pretest counselling, and that NIPT is a screening test and management decisions should not be based on the results of the test alone. ${ }^{[9,11-13]}$

\section{Objective}

While the technology for fetal cfDNA testing becomes more refined and there is general acknowledgement of its high detection rate for the common aneuploidies, several recommendations have been made about the place of NIPT in contingency screening, in both the first and second trimesters. The objective of this study was therefore to determine the value and role of NIPT in a select South African (SA) population.

\section{Methods}

This was a retrospective record review of 82 patients who elected to have NIPT, using cfDNA, between 1 October 2013 and 30 June 2015 at the Morningside Mediclinic Maternal and Fetal Medicine Centre in Johannesburg, SA. The unit is a large referral centre for patients accessing private healthcare, and services offered include combined first-trimester screening (CFTS), second-trimester anomaly ultrasound scans, invasive diagnostic and therapeutic procedures, and follow-up ultrasound scans as indicated. CFTS incorporates maternal characteristics, a first-trimester nuchal translucency ultrasound scan, and serum biochemical investigations (measurement of serum free beta human chorionic gonadotrophin and pregnancy-associated plasma protein A). The Fetal Medicine Foundation database is used for risk calculation in the first and second trimesters. All patients are counselled by the fetal medicine specialist and a genetic counsellor 
about the limitations of screening tests, and based on the risk assessment, elect to have no further tests or procedures, NIPT and/ or an invasive diagnostic test. Obstetric care of the patients is provided by the referring clinicians.

The NIPT, with cfDNA testing, was done in either the first or second trimester using the Natera Panorama test for all the specimens. The Natera Panorama test reports on the fetal fraction of cfDNA, and does a risk calculation for the common trisomies - 21, 18 and 13, monosomy $\mathrm{X}$ and triploidy. Patient records were reviewed to assess the risk profile of patients who elected to have the test, and also to evaluate the results of the NIPT. Patients were contacted telephonically to determine the pregnancy outcomes.

Data were collected on the following parameters: demographics; details of the first- and/or second-trimester screening; results of the NIPT; any invasive tests done; and decisions made about the pregnancy in the event of abnormal test results. Study data were collected and managed using REDCap $^{[14]}$ electronic data capture tools hosted at the University of the Witwatersrand, Johannesburg. JMP, version 11 (SAS Institute, USA) was used for statistical analysis. The study was approved by the University of the Witwatersrand's Human Research Ethics Committee (protocol no. M150935).

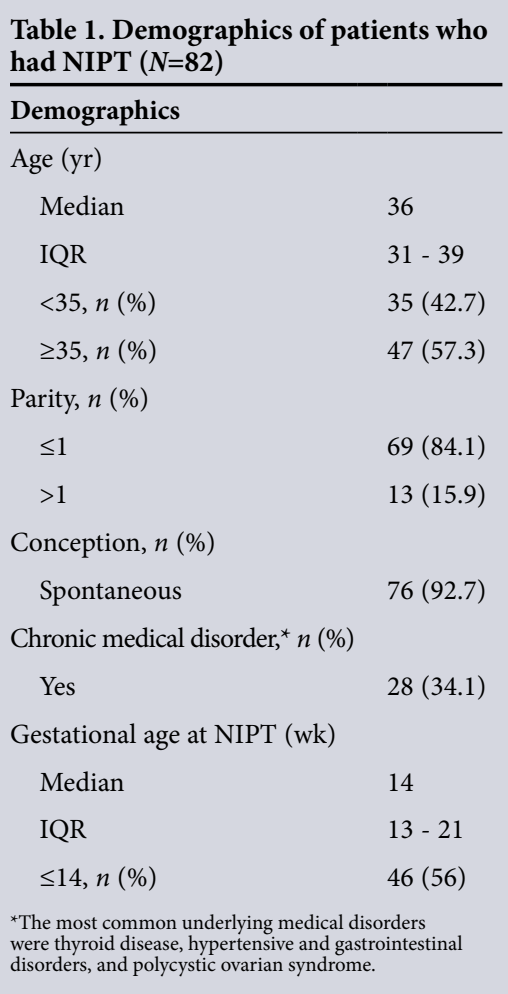

\section{Results}

During the study period, a total of 3473 first- and second-trimester fetal assessments were done at the Morningside Centre, and of these patients, $82(2.3 \%)$ elected to have NIPT. All had singleton pregnancies. The median age of the 82 patients was 36 years (interquartile range (IQR) 31 - 39) (Table 1), and the median weight $(n=81)$ was $62.0 \mathrm{~kg}$ (IQR 58.0 - 72.0). In $92.7 \%$ $(76 / 82)$ of the cases, conception had been spontaneous. Overall, 28 patients $(34.1 \%)$ had an underlying chronic medical disorder, most commonly thyroid disease, hypertensive disorders and polycystic ovarian syndrome.

A total of 67 patients $(81.7 \%)$ had CFTS. The background risk for trisomy 21 was high in $32.8 \%(22 / 67)$ of the patients, while $67.2 \%(45 / 67)$ were in the intermediate-risk group. The patients were assessed as screening positive on CFTS if the adjusted risk was either intermediate $(1: 101-1: 1000)$ or high risk $(>1: 100)$ for the three trisomies $-21,18$ and 13 . Of the patients who had CFTS, 44/67 (65.7\%) screened positive for trisomy 21 , with $9.0 \%$ (6/67) having a high adjusted risk (Table 2). For trisomy 18 and trisomy 13, 9\% (6/67) screened positive in each group. Of the 67 patients who had CFTS, 52 (77.6\%) also had a second-trimester anomaly ultrasound scan. There were 14 patients who only had a second-trimester ultrasound scan. Of the total number of second-trimester ultrasound scans done $(n=66), 14(21.2 \%)$ detected a variety of markers of aneuploidy. Eight of these patients only had a secondtrimester ultrasound scan, and 6 had prior CFTS.

Overall, 82 NIPTs were done, with a median gestational age at the time of the test of 14 weeks (IQR $13-21$ ); a total of 46 patients $(56.1 \%)$ had NIPT at a gestational age of $\leq 14$ weeks. The majority of patients elected to have NIPT based on positive screening on CFTS, or markers of aneuploidy detected on the secondtrimester ultrasound scan (Table 2). Of the tests done, $80 / 82$ (97.6\%) produced results, and the median fetal fraction of cfDNA was $10.5 \%$ (IQR 7.9 - 13.7). Of those with no results, one did not meet quality metrics and the other had a low fetal fraction of cfDNA; both were done in the first trimester and both pregnancies were a result of assisted reproduction. In the case where the specimen did not meet quality metrics, the fetal fraction of cfDNA was $4.8 \%$. Anomalies suggestive of aneuploidy were detected on the firsttrimester ultrasound scan, and the patient elected to have a termination of pregnancy based on the ultrasound findings. There were no karyotype results available to confirm the suspicion of a chromosomal abnormality. In the second case with no NIPT result, the fetal fraction of cfDNA was $3.3 \%$. Increased nuchal translucency was detected on the first-trimester ultrasound scan, and the patient declined invasive testing. She subsequently had a spontaneous miscarriage, and karyotyping of the products of conception confirmed trisomy 18.

Of the 80 NIPT results that were reported, $79(98.8 \%)$ indicated a low risk of trisomy 21 and 18. For trisomy 13, 78/80 cases screened low risk. There were two abnormal NIPT results: one was reported as high risk for trisomy 13 and the other as a triploidy (Table 3). In the first case, a cleft palate was detected on the firsttrimester ultrasound scan, and the adjusted risk on CFTS was intermediate for all the three common trisomies. Trisomy 13 was confirmed on karyotyping of the products of conception after the patient elected to

Table 2. Indications for NIPT $(N=82)$

\begin{tabular}{|c|c|c|c|}
\hline \multicolumn{4}{|l|}{ Screening with CFTS ( $n=67), n(\%)$} \\
\hline & Low risk & Intermediate risk & High risk \\
\hline Trisomy 21 & $23(34.3)$ & $38(56.7)$ & $6(9.0)$ \\
\hline Trisomy 18 & $61(91.0)$ & $5(7.5)$ & $1(1.5)$ \\
\hline Trisomy 13 & $61(91.0)$ & $3(4.5)$ & $3(4.5)$ \\
\hline \multicolumn{4}{|c|}{ Second-trimester ultrasound findings $(n=14),{ }^{*} n(\%)$} \\
\hline Marker(s) of aneuploidy detected & $8(57.1)$ & & \\
\hline No marker(s) detected & $6(42.9)$ & & \\
\hline \multicolumn{4}{|l|}{ Other $^{\dagger}$} \\
\hline Increased background risk of trisomy 21 & 1 & & \\
\hline
\end{tabular}


Table 3. Results of NIPT screening $(N=82)$

\begin{tabular}{|c|c|c|c|}
\hline NIPT results & & Ultrasound findings & Pregnancy outcomes ${ }^{\star}$ \\
\hline Reported results, $n(\%)$ & $80 / 82(97.6)$ & & \\
\hline \multicolumn{4}{|l|}{ cfDNA, $\%$} \\
\hline Median & 10.5 & & \\
\hline IQR & $7.9-13.7$ & & \\
\hline Cases with no results, $n(\%)$ & $2 / 82(2.4)$ & & \\
\hline $\begin{array}{l}\text { Did not meet quality } \\
\text { matrix }\end{array}$ & 1 (cfDNA 4.8\%) & $\begin{array}{l}\text { Anomalies suggestive } \\
\text { of aneuploidy detected }\end{array}$ & $\begin{array}{l}\text { Termination of pregnancy } \\
\text { No karyotype results }\end{array}$ \\
\hline Low cfDNA & 1 (cfDNA 3.3\%) & $\begin{array}{l}\text { Increased nuchal } \\
\text { translucency }\end{array}$ & $\begin{array}{l}\text { Spontaneous miscarriage } \\
\text { Trisomy } 18 \text { on karyotyping }\end{array}$ \\
\hline Abnormal NIPT results & $2 / 82(2.4)$ & & \\
\hline High risk for trisomy 13 & 1 & Cleft palate & $\begin{array}{l}\text { Termination of pregnancy } \\
\text { Trisomy } 13 \text { on karyotyping }\end{array}$ \\
\hline Triploidy & 1 & Normal & $\begin{array}{l}\text { Termination of pregnancy } \\
\text { Triploidy on karyotyping }\end{array}$ \\
\hline
\end{tabular}

have a termination of pregnancy. In the case of triploidy reported on NIPT, and confirmed on CVS, no suspicious findings were detected on CFTS.

Details on pregnancy outcomes were available for $74 / 82$ patients $(90.2 \%)$. Of these, 93.2\% (69/74) had a live birth, $85.3 \%(58 / 68)$ delivering by caesarean section, the majority (44/58) elective. There were no chromosomal abnormalities reported for the live births. A total of 4 patients had a termination of pregnancy, 3 for confirmed chromosomal abnormalities as detailed above, and 1 for spontaneous rupture of membranes and anhydramnios at an early, non-viable gestation. One patient had a spontaneous miscarriage and the fetus had a confirmed chromosomal abnormality, also described above.

\section{Discussion}

In this select SA population, $2.3 \%$ of patients screened during the study period had NIPT. The study sample had either an intermediate or a high background risk for trisomy 21 , largely based on maternal age. The majority of patients elected to have NIPT, using cfDNA, based on either a positive screening result on CFTS or markers detected on the second-trimester anomaly ultrasound scan. Results of NIPT were reported in $97.6 \%$ of the cases, and there were 2 cases $(2.5 \%)$ that screened positive, 1 for trisomy 13 and 1 for triploidy. Of the 2 cases with no results, 1 was confirmed as trisomy 18 on karyotyping and the second had no karyotype results. There were no reported aneuploidies among the cases with known birth outcomes (90.2\%) at the end of the study.
Patient selection in our study was in line with recommendations in various published guidelines. The International Society of Ultrasound in Obstetrics and Gynecology guidelines acknowledge that adequate data on NIPT exist for high-risk obstetric patients, and its use is therefore recommend further state that NIPT may be offered to patients who are not reassured by an intermediate-risk assessment. ${ }^{[12]}$ The American College of Obstetricians and Gynecologists committee opinion on cfDNA screening for fetal aneuploidy also cautions about the use of cfDNA screening in low-risk populations, as there are limited data on costeffectiveness in this group. ${ }^{[13]}$ There were patients in our study who, after counselling, elected to have NIPT despite a low adjusted risk assessment. In cases where a structural anomaly is detected on ultrasound, the recommendation is that diagnostic testing should be offered instead of cfDNA screening. ${ }^{[13]}$

Overall in our study the performance of NIPT screening was good, in that there were only two cases that had no results. In cases where results are not reported, the recommendation is for patients to be offered detailed ultrasound assessment and diagnostic testing because of an increased risk of aneuploidy. ${ }^{[13]}$ In both cases with no results in our study, patients declined invasive testing despite first-trimester ultrasound findings suggestive of an increased risk of aneuploidy. In the one case that screened positive on NIPT and was confirmed as trisomy 13 on karyotyping, aneuploidy was suspected based on the for this group. ${ }^{[12]}$ However, the guidelines first-trimester ultrasound findings, calling into question the value of cfDNA screening in cases with obvious abnormalities on ultrasound. While patients were counselled about the need for invasive testing following an abnormal NIPT result, or in cases where there were no results, some patients elected to have a termination of pregnancy based on ultrasound and NIPT results alone. Patients were reassured by negative NIPT screening results, and all the patients who screened negative elected not to have an invasive test, even those who had a high adjusted risk on CFTS or had markers detected on the second-trimester ultrasound scan. Regardless of the NIPT results, patients need to be counselled about the limitations of this screening test, and that management decisions cannot be based on NIPT results alone.

The value of NIPT in this select population was that it was possible to avoid a number of invasive tests in the small group with a high adjusted risk for trisomy 21 . As per guidelines, NIPT was not used as a stand-alone test, but rather had a role in contingency screening. However, our study had limitations, in that the sample size was small and it was conducted in a select population, and findings cannot be generalised to a wider population. Also, the pregnancy outcomes were not known in a small proportion of patients, but it is unlikely that aneuploidies would not have been reported if missed. The strengths, however, are that the centre where the study was done uses internationally recognised standards for CFTS and the secondtrimester anomaly ultrasound scan. Also, the same test, the Panorama test, was used for all the specimens and the fetal fraction of cfDNA was reported on routinely. There is certainly a need for larger studies in SA to determine the value and role of NIPT screening, especially in low-risk populations and in cases where obvious anomalies are detected on ultrasound.

\section{Conclusion}

In conclusion, NIPT screening, using cfDNA, should be offered as part of contingency screening in high- and intermediaterisk obstetric patients. Patients should be counselled, ideally by a genetic counsellor before and after the test, about the limitations of NIPT screening and that management decisions should not be made without invasive diagnostic testing when a result is abnormal.

Acknowledgement. We thank Sipelele Mnyani for assisting with the data analysis. 
1. Nicolaides KH, Azar G, Byrne D, Mansur C, Marks K. Fetal nuchal translucency: Ultrasound screening for chromosomal defects in first trimester of pregnancy. BMJ 1992;304(6831):867-869. DOI:10.1136bmj.304.6831.867

2. Snijders RJM, Johnson S, Sebire NJ, Noble PL, Nicolaides KH. First-trimester ultrasound screening for chromosomal defects. Ultrasound Obstet Gynecol 1996-7(3):216-226. DOI:10.1046/j.14690705.1996.07030216.x

3. Wald NJ, Watt HC, Hackshaw AK. Integrated screening for Down's syndrome based on tests performed during the first and second trimesters. N Engl J Med 1999;341(7):461-467. DOI:10.1056 NEJM19990812341070

4. Lo YM, Corbetta N, Chamberlain PF, et al. Presence of fetal DNA in maternal plasma and serum. Lancet 1997;350(9076):485-487. DOI:10.10.1016/S0140-6736(97)02174-0

5. Benn P, Cuckle H, Pergament E. Non-invasive prenatal testing for aneuploidy: Current status and future prospects. Ultrasound Obstet Gynecol 2013;42(1):15-33. DOI:10.1002/uog.12513

6. Poon LC, Musci T, Song K, et al. Maternal plasma cell-free fetal and maternal DNA at 11-13 weeks' gestation: Relation to fetal and maternal characteristics and pregnancy outcomes. Fetal Diagn Ther 2013;33(4):215-223. DOI:10.1159/000346806

7. Martin A, Krishna I, Martina B, et al. Can the quantity of cell-free fetal DNA predict preeclampsia: A systematic review. Prenat Diagn 2014;34(7):685-691. DOI:10.1002/pd.4416

systematic review. Prenat Diagn 2014;34(7).685-69. DOI.10.1002/pd. eclampsia. Ultrasound Obstet Gynecol 2015;45(1):106-111. DOI-10.1002/uog.14671
9. Royal College of Obstetricians and Gynaecologists. Non-invasive Prenatal Testing for Chromosomal Abnormality using Maternal Plasma DNA. Scientific Impact Paper No. 15. London: RCOG, 2014.

10. Thilaganathan B. Ushering in a new dawn in obstetrics and gynecology: The industry of cell-free DNA testing. Ultrasound Obstet Gynecol 2015;45(1):1-3. DOI:10.1002/uog.14744

11. Gregg AR, Gross SJ, Best RG, et al., for the Noninvasive Prenatal Screening Work Group of the American College of Medical Genetics and Genomics. ACMG statement on noninvasive prenatal screening for fetal aneuploidy. Genet Med 2013;15(5):395-398. DOI:10.1038/gim.2013.29

12. Salomon LJ, Alfirevic Z, Audibert F, et al. ISUOG consensus statement on the impact of non-invasive prenatal testing (NIPT) on prenatal ultrasound practice. Ultrasound Obstet Gynecol 2014;44(1):122123. DOI:10.1002/uog.13393

13. American College of Obstetricians and Gynecologists. Cell-free DNA screening for fetal aneuploidy. Committee Opinion No. 640. Obstet Gynecol 2015;126(3):e31-e37. DOI:10.1097/ AOG.0000000000001051

14. Harris PA, Taylor R, Thielke R, Payne J, Conde JG. Research electronic data capture (REDCap) - a metadata-driven methodology and workflow process for providing translational research informatics support. J Biomed Inform 2009;42(2):377-381. DOI:10.1016/j.jbl.2008.08.010

Accepted 4 April 2016 